\title{
A Single Reactive Noncanonical Amino Acid is Able to Dramatically Stabilize Protein Structure
}

\author{
Jack C. $\mathrm{Li}^{\dagger}$, Fariborz Nastertorabi ${ }^{\ddagger}$, Weimin Xuan ${ }^{\dagger}$, Gye Won Han ${ }^{\ddagger}$, Raymond C. Stevens ${ }^{\ddagger}$, \\ Peter G. Schultz ${ }^{\dagger,}{ }^{*}$ \\ †Department of Chemistry and Skaggs Institute for Chemical Biology, The Scripps Research \\ Institute, La Jolla, California 92037, United States \\ ¥Department of Biological Sciences, Bridge Institute, Michaelson Center for Convergent \\ Bioscience, University of Southern California, Los Angeles, California 90089, United States
}

\section{Abstract}

\begin{abstract}
A p-isothiocyanate phenylalanine mutant of the homodimeric protein homoserine osuccinyltransferase (MetA) was isolated in a temperature dependent selection from a library of $m e t A$ mutants containing noncanonical amino acids. This mutant protein has a dramatic increase of $24^{\circ} \mathrm{C}$ in thermal stability compared to the wild type protein. Peptide mapping experiments revealed that the isothiocyanate group forms a thiourea crosslink to the $\mathrm{N}$ terminal proline of the other monomer, despite the two positions being > $30 \AA$ apart in the X-ray crystal structure of the wild type protein. These results show that an expanded set of building blocks beyond the canonical 20 amino acids can lead to significant changes in the properties of proteins.
\end{abstract}

\begin{abstract}
We and others have shown that genetically encoded noncanonical amino acids (ncAAs) can be used to generate proteins with new or enhanced properties ${ }^{1-10}$. A powerful approach for identifying such mutants involves genetic screens or selections using libraries of random protein variants containing ncAAs ${ }^{11}, 12$. The latter have been used to identify proteins with enhanced properties, including mutations that increase catalytic activity and enhance protein thermal stability ${ }^{13}$. Here, we use a temperature dependent selection to identify a single mutation of Phe264 to p-isothiocyanate phenylalalnine (pNCSF) on the surface of homodimeric $E$. coli homoserine o-succinyltransferase that is able to form a covalent crosslink with the distant $\mathrm{N}$-terminal Pro of the other monomer, and in doing so significantly stabilizes MetA.

MetA is an essential enzyme in E. coli involved in methionine biosynthesis that exists naturally as a homodimer. It has been previously shown that the growth of $E$. coli is limited at temperatures above $37^{\circ} \mathrm{C}$ in part due to the relative instability of MetA at these higher temperatures ${ }^{14}$. As a result, E. coli containing more thermostable forms of MetA exhibit
\end{abstract}

\footnotetext{
*Corresponding Author: schultz@ scripps.edu.

Supporting Information

The Supporting Information is available free of charge on the ACS Publications website.

Supplementary figures (S1-S6), strains and plasmids used, experimental methods, supplementary references

The authors declare no competing financial interests.
} 
increased growth rates at $44^{\circ} \mathrm{C}^{15}, 16$. We have been able to exploit this property to screen for mutants of MetA containing ncAAs that stabilize MetA at elevated temperatures and thus confer a competitive growth advantage compared to wild-type MetA. In particular we identified a p-benzoyl phenylalanine mutant that stabilized MetA by $21^{\circ} \mathrm{C}$ by forming a reversible covalent crosslink with a cysteine in the other subunit of the homodimer ${ }^{13}$. Given this unexpected finding we carried out the same selection using the electrophilic amino acid pNCSF, which has been previously shown to form thiourea crosslinks with amines both intra and intermolecularly under native conditions while being stable to protein isolation conditions ${ }^{17}$.

To carry out this selection, a comprehensive amber nonsense TAG scanning library (containing 261 mutants out of 308 positions) of MetA mutants was transformed into E. coli JW3973-1 ( $\triangle m e t A)$ along with a second plasmid containing an orthogonal tRNA/ aminoacyl-tRNA synthetase (pUltra-CNFRS ${ }^{18}$ ) pair that is able to selectively and efficiently incorporate pNCSF at TAG sites within the protein. Transformants were grown at $44^{\circ} \mathrm{C}$ in liquid minimal media containing $0.5 \mathrm{mM}$ pNCSF for $20-40$ hours, which has previously been demonstrated to be an effective condition for obtaining mutants which can outperform WT MetA at elevated temperatures. Cultures were subsequently plated and colonies were picked and sequenced, with a number of mutants being then recombinantly expressed for biochemical testing.

Among the mutants obtained was F264pNCSF. We expressed and purified the C-terminal 6xHis tagged variants of WT and F264pNCSF MetA (hereafter referred to as WT MetA and F264pNCSF MetA) in yields of roughly $8-9 \mathrm{mg} / \mathrm{mL}$ and $3-4 \mathrm{mg} / \mathrm{mL}$, respectively. The dimeric nature of both the WT and F264pNCSF MetA was verified by size exclusion chromatography (Figure S1) and mass spectrometry (Figure S7). Analysis of the MetA mutant at RT by denaturing polyacrylamide gel electrophoresis (SDS-PAGE) did not show increased mobility relative to WT MetA, consistent with a free isothiocyanate group in F264pNCCSF MetA and the absence of either intramolecular or intermolecular covalent crosslinks ${ }^{19,20}$ (Figure S6). As expected, the isothiocyanate group of F264pNCSF MetA can be rapidly labeled at room temperature (RT) using ethanolamine to irreversibly form the hydroxyethyl thiourea, which is readily detectable as a 61 Da increase by mass spectrometry. However, when the mutant protein is first incubated at $55^{\circ} \mathrm{C}$ prior to exposure to ethanolamine (500 eq. in $100 \mathrm{mM}$ sodium bicarbonate, $\mathrm{pH} 8.5$ ), dramatically reduced labeling is detected, suggesting a heat induced intra- or intermolecular reaction of the isothiocyanate functionality (Figure S3).

Surprisingly, when the protein melting curve was quantified by temperature dependent circular dichroism spectroscopy (CD), F264pNCSF MetA showed a dramatic $24^{\circ} \mathrm{C}$ increase from $52^{\circ} \mathrm{C}$ to $\sim 76^{\circ} \mathrm{C}$ in melting temperature $\left(\mathrm{T}_{\mathrm{m}}\right.$ ) compared to the WT protein, including after pre-heating of the sample to $50^{\circ} \mathrm{C}$ (Figure $1 \mathrm{~b}$ ). We speculated that the isothiocyanate group may be reacting during thermal scanning itself, and in fact found that upon incubating the recombinant F264pNCSF MetA either briefly at $50^{\circ} \mathrm{C}$ or overnight at $37^{\circ} \mathrm{C}$ in DPBS, a significant dimeric fraction appeared on SDS-PAGE (Figure 1a) and by mass spectrometry (Figure S6) although we were unable to isolate the covalent dimer as denaturation is required to avoid co-purification with the native dimer and we have not been able to identify 
suitable refolding conditions. It is possible that upon heating, we are stabilizing the native protein or a folding intermediate leading to an increased apparent melting temperature. To begin to distinguish these possibilities, the catalytic activity was measured for the WT and F264pNCSF proteins as a function of temperature. It was found that F264pNCSF MetA had roughly half the $\mathrm{k}_{\text {cat }}\left(21 \mathrm{~s}^{-1}\right)$ of the WT protein $\left(39 \mathrm{~s}^{-1}\right)$. However, whereas WT activity sharply decreased to near zero at temperatures of $50^{\circ} \mathrm{C}$ and above, the mutant protein retained $>60 \%$ activity through $60^{\circ} \mathrm{C}$ (Figure S2) with thermal inactivation roughly paralleling the $\mathrm{CD}$ melting curves. This result suggests that we are stabilizing a near native active site conformation, or a partially unfolded protein with very significant catalytic activity.

To further confirm that pNCSF is forming a covalent crosslink upon heating, we sought to isolate the crosslinked peptide by proteolysis followed by mass spectrometry (MS) analysis and tandem mass spectrometry (MS/MS). A concentrated sample of F264pNCSF MetA (25 $\mathrm{mg} / \mathrm{mL}$ ) in phosphate buffered saline (DPBS) was briefly incubated from $30^{\circ} \mathrm{C}$ to $50^{\circ} \mathrm{C}$ incrementally, before being proteolyzed by trypsin overnight and then analyzed by MS. As expected, as the initial incubation temperature was increased the ratio of crosslinked peptide to uncrosslinked peptide increased substantially from 1.2 to 4.8 (Figure S2). The trypsin digested crosslinked peptide ion was further subjected to MS/MS, which confirmed a thiourea crosslink between Pro 2 and pNCSF 264 (Figure 2, S4).

We attempted to obtain a crystal structure of the crosslinked protein. Unfortunately, no suitable crystals of F264pNCSF MetA could be obtained. However, we were able to crystallize WT MetA (after truncation of the C-terminus residues 297-309 and a single P257I point mutation was introduced), and the X-ray crystal structure of WT MetA was determined at $<1.9 \AA$ (Figure 3). The structure shows that MetA exhibits domain swapping through predominantly an N-terminal alpha helix, which participates in a helix-helix interaction with residues $48-56$ of the opposing monomer and likely contributes to protein stabilization. The N-terminal region is connected to the rest of the protein through a highly disordered loop. Most surprisingly however, we found that the $\mathrm{N}$-terminal proline was in fact very far from Phe264 (measuring more than $30 \AA$ by linear distance) and largely buried underneath surrounding loops. Similarly, Phe264, which is mutated to pNCSF in the MetA mutant, is likewise partially buried beneath multiple adjacent amino acids in the WT protein, although the isothiocyanate group may disrupt structure in the mutant protein. From this, we can conclude that this $\mathrm{N}$-terminal domain must exhibit a high degree of conformational flexibility and the region surrounding Phe 264 must be similarly flexible. This provides an explanation as to why no initial crosslinking was observed, as the recombinant protein is expressed at and subsequently purified at $<24^{\circ} \mathrm{C}$ in which it is largely in an unreactive conformation.

To explore the conformational flexibility of the $\mathrm{N}$-terminal domain, temperature dependent fluorescence polarization measurements were performed. To this end, p-azidophenylalanine (pAzF) was substituted for the solvent accessible residue, Arg 4, and subsequently conjugated to an Alexa Fluor 488 sDIBO alkyne (Thermo) by a click reaction. Separately, Glu169, located on a surface exposed region of the protein, was similarly replaced with $\mathrm{pAzF}$ and fluorescently labeled. Fluorescence polarization experiments revealed that 
fluorescence anisotropy decreased at both sites with increasing temperature from $18^{\circ} \mathrm{C}$ to $36^{\circ} \mathrm{C}$ (Figure S5). However, above $36^{\circ} \mathrm{C}$ the fluorescence anisotropy at residue 169 increased, possibly due to the protein core beginning to denature and aggregate at these temperatures. These results are consistent with a somewhat flexible $\mathrm{N}$-terminus that can crosslink to pNCSF 264 in the mutant protein as temperature is increased, resulting in increased protein stability.

Single mutations in proteins can often have dramatic effects on their activity and stability, especially when attempting to reduce either. Previous efforts to increase protein stability however have been much more limited in their success, with mutagenesis attempts rarely being able to increase $T_{m}$ by more than a few degrees, unless it is the reversion of a previous destabilizing mutation or the additive effects of a series of beneficial changes ${ }^{21}$, even when covalent bonds in the form of disulfide bonds are being formed ${ }^{22}$. A notable exception is when we previously showed that the incorporation of the $p$-benzoyl phenylalanine ncAA into MetA was able to increase $\mathrm{T}_{\mathrm{m}}$ by $21^{\circ} \mathrm{C}$ through the formation of a hemithioketal with a surface cysteine by reversible covalent crosslinking of the two monomers. Here, we further show how the incorporation of a single ncAA, pNCSF, which has the potential to readily form covalent thiourea crosslinks with nearby amines, can dramatically alter stability of a protein - in this case of MetA by $24^{\circ} \mathrm{C}$. We hypothesize that pNCSF is able to irreversibly crosslink two distal sites that are likely conformationally flexible, resulting in a covalently trapped dimer that results in a large increase in $\mathrm{T}_{\mathrm{m}}{ }^{22}$. In this way, we continue to show how utilization of an expanded chemical space can lead to unexpected and dramatic changes in protein structure and function.

\section{Supplementary Material}

Refer to Web version on PubMed Central for supplementary material.

\section{ACKNOWLEDGMENT}

The authors thank the Scripps Research Institute Mass Spectrometry Core, L. Hoang, the Ian Wilson Lab, H. Tien, D. Ashok and E. Bentley for their valuable experimental assistance. This work is supported by NIH grant R01 GM062159.

\section{REFERENCES}

(1). Xiang Z, Lacey VK, Ren H, Xu J, Burban DJ, Jennings PA, and Wang L (2014) Proximity-enabled protein crosslinking through genetically encoding haloalkane unnatural amino acids, Angew Chem Int Ed Engl, 2190-2193.

(2). Xie J, and Schultz PG (2006) A chemical toolkit for proteins — an expanded genetic code, Nature Reviews, 775-782.

(3). Luo X, Fu G, Wang R, Zhu X, Zambaldo C, Liu R, Liu T, Lyu X, Du J, Xuan W, Yao A, Reed SA, Kang M, Zhang Y, Guo H, Huang C, Yang P, Wilson IA, Schultz PG, and Wang F (2017) Genetically encoding phosphotyrosine and its nonhydrolyzable analog in bacteria, Nat Chem Bio, 845-849. [PubMed: 28604693]

(4). Brustad E, Bushey ML, Lee JW, Groff D, Liu W, and Schultz PG (2008) A genetically encoded boronate-containing amino acid, Angew Chem Int Ed Engl, 8220-8223. [PubMed: 18816552]

(5). Chin JW, Santoro SW, Martin AB, King DS, Wang L, and Schultz PG (2002) Addition of p-AzidoL-phenylalanine to the Genetic Code of Escherchia coli, J Am Chem Soc, 9026-9027. [PubMed: 12148987] 
(6). Deiters A, and Schultz PG (2005) In vivo incorporation of an alkyne into proteins in Escherichia coli, Bioorg Med Chem Lett, 1521-1524. [PubMed: 15713420]

(7). Tippmann EM, Liu W, Summerer D, Mack AV, and Schultz PG (2007) A genetically encoded diazirine photocrosslinker in Escherichia coli, Chembiochem, 2210-2214. [PubMed: 18000916]

(8). Furman JL, Kang M, Choi S, Cao Y, Wold ED, Sun SB, Smider VV, Schultz PG, and Kim CH (2014) A genetically encoded aza-Michael acceptor for covalent cross-linking of protein-receptor complexes, J Am Chem Soc, 8411-8417. [PubMed: 24846839]

(9). Wang L, and Schultz PG (2004) Expanding the genetic code, Angew Chem Int Ed Engl, 34-66. [PubMed: 15599909]

(10). Wang L, Xie J, and Schultz PG (2006) Expanding the genetic code, Annu Rev Biophys Biomol Struct, 225-249. [PubMed: 16689635]

(11). Liu T, Wang Y, Luo X, Li J, Reed SA, Xiao H, Young TS, and Schultz PG (2016) Enhancing protein stability with extended disulfide bonds, Proc Natl Acad Sci U S A, 5910-5915. [PubMed: 27162342]

(12). Xiao H, Nasertorabi F, Choi SH, Han GW, Reed SA, Stevens RC, and Schultz PG (2015) Exploring the potential impact of an expanded genetic code on protein function, Proc Natl Acad Sci U S A, 6961-6966. [PubMed: 26038548]

(13). Li JC, Liu T, Wang Y, Mehta AP, and Schultz PG (2018) Enhancing Protein Stability with Genetically Encoded Noncanonical Amino Acids, J Am Chem Soc, 15997-16000. [PubMed: 30433771]

(14). Ron EZ, and Shani M (1971) Growth Rate of Escherichia coli at Elevated Temperatures: Reversible Inhibition of Homoserine Trans-Succinylase, Journal of Bacteriology, 397-400. [PubMed: 4939759]

(15). Mordukhova EA, Kim D, and Pan JG (2013) Stabilized homoserine o-succinyltransferases (MetA) or L-methionine partially recovers the growth defect in Escherichia coli lacking ATPdependent proteases or the DnaK chaperone, BMC Microbiol, 179.

(16). Mordukhova EA, Lee HS, and Pan JG (2008) Improved thermostability and acetic acid tolerance of Escherichia coli via directed evolution of homoserine o-succinyltransferase, Appl Environ Microbiol, 7660-7668. [PubMed: 18978085]

(17). Xuan W, Li J, Luo X, and Schultz PG (2016) Genetic Incorporation of a Reactive Isothiocyanate Group into Proteins, Angew Chem Int Ed Engl, 10065-10068. [PubMed: 27418387]

(18). Young DD, Young TS, Jahnz M, Ahmad I, Spraggon G, and Schultz PG (2011) An evolved aminoacyl-tRNA synthetase with atypical polysubstrate specificity, Biochemistry, 1894-1900. [PubMed: 21280675]

(19). Dunker AK, and Kenyon AJ (1976) Mobility of Sodium Dodecyl Sulphate Protein Complexes, Biochemical Journal, 191-197.

(20). Rath A, Glibowicka M, Nadeau VG, Chen G, and Deber CM (2009) Detergent binding explains anomalous SDS PAGE migration of membrane proteins, Proc Natl Acad Sci U S A, 1760-1765. [PubMed: 19181854]

(21). Dumon C, Varvak A, Wall MA, Flint JE, Lewis RJ, Lakey JH, Morland C, Luginbuhl P, Healey S, Todaro T, DeSantis G, Sun M, Parra-Gessert L, Tan X, Weiner DP, and Gilbert HJ (2008) Engineering hyperthermostability into a GH11 xylanase is mediated by subtle changes to protein structure, J Biol Chem, 22557-22564. [PubMed: 18515360]

(22). Dombkowski AA, Sultana KZ, and Craig DB (2014) Protein disulfide engineering, FEBS Lett, 206-212. [PubMed: 24291258] 
a).

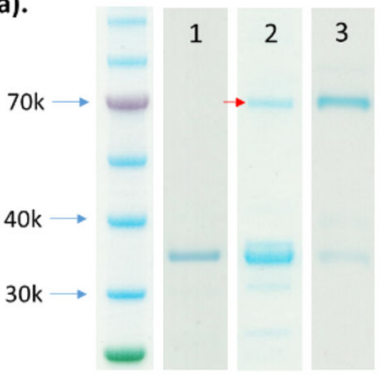

b).

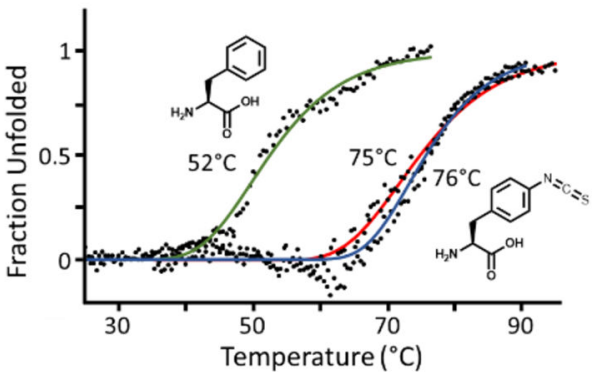

Figure 1.

(a) SDS-PAGE of F264pNCSF MetA. Lane 1: untreated; Lane 2: after heating at $50^{\circ} \mathrm{C}$ for $10 \mathrm{~min}$ in DPBS, $\mathrm{pH}$ 7.4. Lane 3: after heating from $20^{\circ} \mathrm{C}$ to $50^{\circ} \mathrm{C}$ at $0.5^{\circ} \mathrm{C} / \mathrm{min}$ in $200 \mathrm{mM}$ sodium phosphate, $\mathrm{pH}$ 7.4. (b) CD melting curves of WT MetA (green) and F264pNCSF MetA (blue), and F264pNCSF MetA after first being heated to $50^{\circ} \mathrm{C}$ (red) in $200 \mathrm{mM}$ sodium phosphate, $\mathrm{pH}$ 7.4. The $\mathrm{T}_{\mathrm{m}}$ values were estimated based on the maximum slope. 


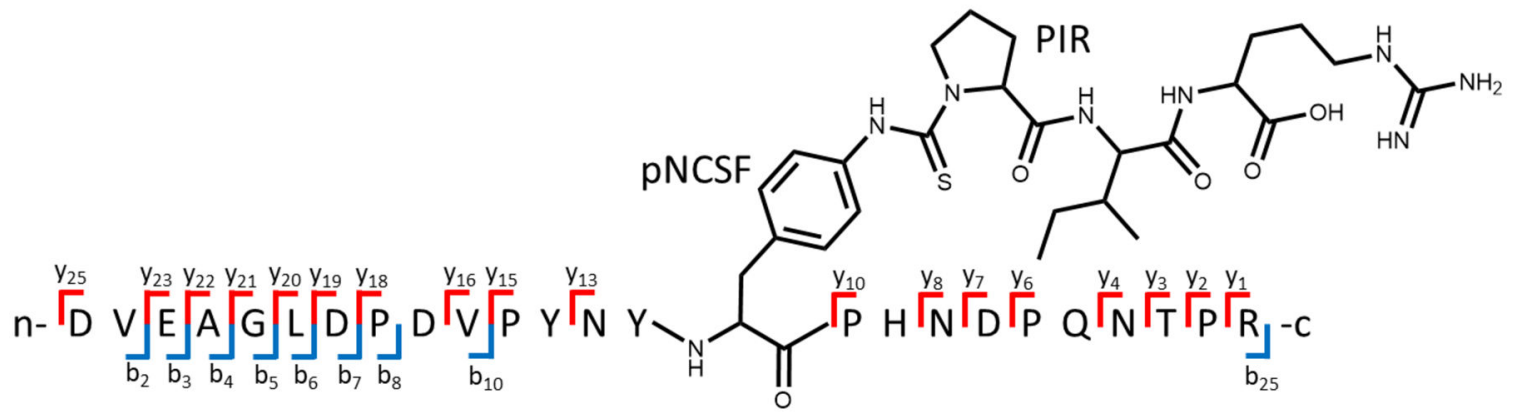

Figure 2.

The expected thiourea crosslink between pNCSF264 and Pro2 after trypsin digestion. Fragments identified from MS/MS analysis of the digested peptide are indicated, and confirm the thiourea crosslink. 


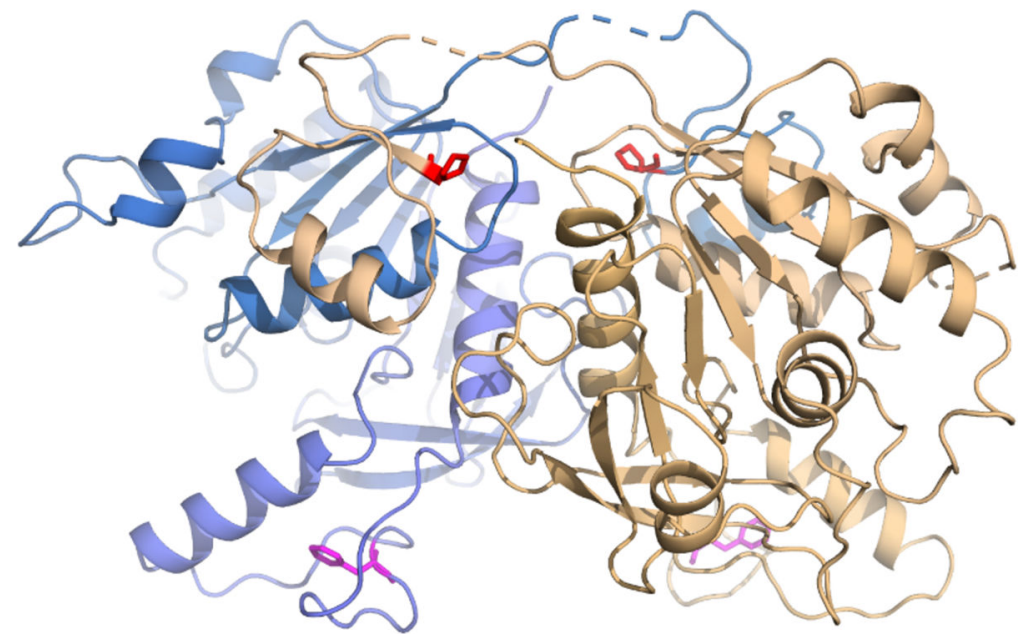

Figure 3.

Crystal structure of WT MetA (PDB code 6MTG). The two monomeric units are colored in beige and blue. Phe264, which is mutated to pNCSF in the mutant MetA and is crosslinked as a thiourea, is colored magenta and Pro2 is colored red. 